\title{
Price Prediction of Share Market using Artificial Neural Network (ANN)
}

\author{
Zabir Haider Khan \\ Department of CSE, SUST, \\ Sylhet, Bangladesh.
}

\author{
Tasnim Sharmin Alin \\ Department of CSE, SUST, \\ Sylhet, Bangladesh.
}

\author{
Md. Akter Hussain \\ Department of CSE, SUST, \\ Sylhet, Bangladesh.
}

\begin{abstract}
Share Market is an untidy place for predicting since there are no significant rules to estimate or predict the price of share in the share market. Many methods like technical analysis, fundamental analysis, time series analysis and statistical analysis etc are all used to attempt to predict the price in the share market but none of these methods are proved as a consistently acceptable prediction tool. Artificial Neural Network (ANN), a field of Artificial Intelligence (AI), is a popular way to identify unknown and hidden patterns in data which is suitable for share market prediction. For predicting of share price using ANN, there are two modules, one is training session and other is predicting price based on previously trained data. We used Backpropagation algorithm for training session and Multilayer Feedforward network as a network model for predicting price. In this paper, we introduce a method which can predict share market price using Backpropagation algorithm and Multilayer Feedforward network.
\end{abstract}

\section{General Terms}

Artificial Neural Network, Machine Learning, Back propagation Algorithms, Share Market.

\section{Keywords}

Artificial Neural Network (ANN), Prediction, Artificial

Intelligence (AI), Backpropagation (BP), Multilayer

Feedforward Network, Neural Network (NN).

\section{INTRODUCTION}

A share market is a place of high interest to the investors as it presents them with an opportunity to benefit financially by investing their resources on shares and derivatives of various companies. It is a chaos system; meaning the behavioral traits of share prices are unpredictable and uncertain. To make some sort of sense of this chaotic behavior, researchers were forced to find a technique which can estimate the effect of this uncertainty to the flow of share prices. From the analyses of various statistical models, Artificial Neural Networks are analogous to nonparametric, nonlinear, regression models. So, Artificial Neural Networks (ANN) certainly has the potential to distinguish unknown and hidden patterns in data which can be very effective for share market prediction. If successful, this can be beneficial for investors and financers and that can positively contribute to the economy.
There are different methods that have been applied in order to predict Share Market returns. Tang and Fishwick[1]; Wang and Leu [2] provided a general introduction of how a neural network should be developed to model financial and economic time series. During the last decade, Artificial Neural Networks have been used in share market prediction. One of the first such projects was by Kimoto et al. [3] who had used ANN for the prediction of Tokyo stock exchange index. Minzuno et al. [4] applied ANN again to Tokyo stock exchange to predict buying and selling signals with an overall prediction rate of $63 \%$. Sexton et al. [5] theorized that the use of momentum and start of learning at random points may solve the problems that may occur in training process in 1998. Phua et al. [6] applied neural network with genetic algorithm to the stock exchange market of Singapore and predicted the market direction with an accuracy of $81 \%$.

This paper demonstrates Back propagation method for training the Neural Network and Multilayer Feed forward network in order to forecast the share values. The aim of this paper is to use ANNs to forecast Bangladesh Stock Exchange market index values with reasonable a degree of accuracy.

\section{PREDICTION METHOD ANALYSIS:}

Trading shares and commodities were primarily based on intuitions. As the trading grew, people tried to find methods and tools which can accurately predict the share prices increasing their gains and minimizing their risk. Many methods like fundamental analysis, technical analysis, and machine learning method have all been used to attempt predictions of share prices but none of these methods have been proven as a consistently applicable prediction tool.

\subsection{Fundamental Analysis}

Fundamental analysis is the physical study of a company in terms of its product sales, manpower, quality, infrastructure etc. to understand it standing in the market and thereby its profitability as an investment [7]. The fundamental analysts believe that the market is defined 90 percent by logical and 10 percent by physiological factors. But, this analysis is not suitable for our study because the data it uses to determine the intrinsic value of an asset does not change on daily basis and therefore is not suitable for short-term basis. However, this analysis is suitable for predicting the share market only in long-term basis. 


\subsection{Technical Analysis}

The technical analysis predicts the appropriate time to buy or sell a share. Technical analysts use charts which contain technical data like price, volume, highest and lowest prices per trading to predict future share movements. Price charts are used to recognize trends. These trends are understood by supply and demand issues that often have cyclical or some sort of noticeable patterns. To understand a company and its profitability through its share prices in the market, some parameters can guide an investor towards making a careful decision. These parameters are termed Indicators and Oscillators [7]. This is a very popular approach used to predict the market. But the problem of this analysis is that the extraction of trading rules from the study of charts is highly subjective, as a result different analysts extract different trading rules studying the same charts. This analysis can be used to predict the market price on daily basis but we will not use this approach because of its subjective nature.

\subsection{Machine Learning Methods}

Machine learning approach is attractive for artificial intelligence since it is based on the principle of learning from training and experience. Connectionist models [8] such as ANNs are well suited for machine learning where connection weights adjusted to improve the performance of a network.

\section{CHALLENGE IN PREDICTION OF SHARE MARKET PRICE}

The main problem in predicting share market is that the share market is a chaos system. There are many variables that could affect the share market directly or indirectly. There are no significant relations between the variables and the price. We cannot draw any mathematical relation among the variables. There are no laws of predicting the share price using these variables.

\section{OUR SYSTEM ARCHITECTURE}

For this kind of chaotic system the neural network approach is suitable because we do not have to understand the solution. This is a major advantage of neural network approaches [9]. On the other hand in the traditional techniques we must understand the inputs, the algorithms and the outputs in great detail. With the neural network we just need to simply show the correct output for the given inputs. With sufficient amount of training, the network will mimic the function $[9,10]$. Another advantage of neural network is that during the tanning process, the network will learn to ignore any inputs that don't contribute to the output $[9,10]$.

For our system, there is a training phase where some parameters named weights are found from this section and Backpropagation Algorithm is used for this training phase. These weights are used in prediction phase using same equations which are used in training phase. This is our basic Architecture of our System and this approach is known as a Feedforward Network.. There are a lot of inputs in share market which are impacts in share price. But all the inputs are not used in our system because their impact are not significant in share market price. We used 5 inputs for the system. The inputs are: General Index (GI), P/E ratio, Net Asset Value (NAV), Earnings per Share (EPS) and volume. Then we normalized the data set according to the network and the feed the data to the network.

\subsection{Backpropagation with Feedforeword NN}

Back-propagation algorithm $[8,11,14]$ is basically the process of back-propagating the errors from the output layers towards the input layer during training sessions. Back-propagation is necessary because the hidden units have no target values which can be used, so these units must be trained based on errors from the previous layers. The output layer has a target value which is used to compare with calculated value. As the errors are backpropagated through the nodes, the connection weights are continuously updated. Training will occur until the errors in the weights are adequately small to be accepted. On the other hand the computational complexity of Backpropagation Algorithm is only $\mathrm{O}(\mathrm{n})$. These features of the algorithm are the main criteria for predicting share prices accurately.

The main steps using the Backpropagation algorithm as follows:

Step 1: Feed the normalized input data sample, compute the corresponding output;

Step 2: Compute the error between the output(s) and the actual target(s);

Step 3: The connection weights and membership functions are adjusted;

Step 4: IF Error > Tolerance THEN go to Step 1 ELSE stop.

\section{MODEL ANALYSIS}

We used feedforward neural network which has a input layer with 5 neurons, a hidden layer which has 5 neurons and a output layer with single neuron. The backpropagation algorithm has been used for training the network.

\subsection{Training Phase}

There are two phases $1^{\text {st }}$ is the training phase and $2^{\text {nd }}$ is the prediction phase. The training phase can be divided into two parts, the propagation phase and the weight update phase.

In the propagation phase $1^{\text {st }}$ the input data is normalized for feeding the network into the input nodes using the formula:

$$
v^{\prime}=\frac{v-\min A}{\operatorname{maxA} A \min A}\left(n e w_{\max A}-n e w_{\min A}\right)+n e w_{\min A}
$$

Here,

$$
\mathrm{V}^{\prime}=\text { Normalized Input. }
$$$$
\mathrm{V}=\text { Actual Input. }
$$

Min A, Max A = Boundary values of the old data range. $\mathrm{New}_{\min \mathrm{A}}$, New $\operatorname{maxA}=$ Boundary values of the new data range. In this case it is -1 and 1 because the backpropagation can only handle data between -one to one.

[12] 


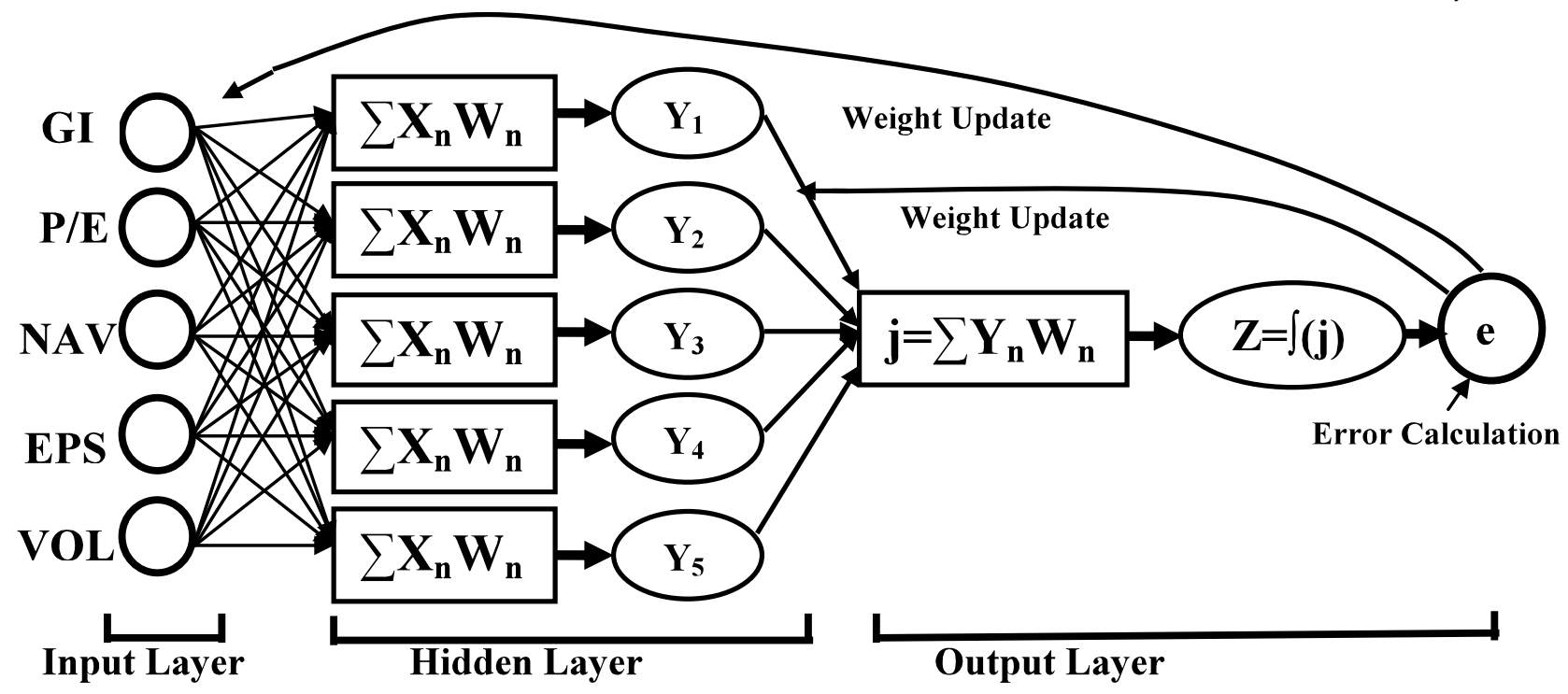

Fig 1: Training phase

From the figure 1 we can see that, the normalized input data are fed into the input layer, then the weights are multiplied with the each input data and enter into the neurons of hidden layer, the function of a single neuron are described in the figure 2 , in our model we used single hidden layer. In our model the hidden layer neurons has the same functions as the input layers neurons. After that each neuron passes the output to the next neuron of the output layer. The output layer calculate the in the same way as the hidden layer neuron and generate the final out put which is the compared with the real output and calculate an error signal 'e'.

$$
e=\frac{\text { actual output }- \text { ANN output }}{\text { actual output }}
$$

The error ' $\mathrm{e}$ ' is generated from the Propagation Phase is used to update the weight using the following formula:

Updated Weight $=$ weight $($ old $)+$ learning rate $*$ output error $*$ output(neurons $\mathrm{i}) * \operatorname{output}($ neurons $\mathrm{i}+1) *$

(1 - Output (neurons $\mathrm{i}+1)$ ).

The above process is done in every weight matrix in the network for updating weight .The Phase 1 and Phase 2 procedure repeatedly used until the sum of square error is zero or close to zero.

Like the figure 2 each neuron is composed of two units. First unit adds products of weights coefficients and input signals [12]. Then this output enter into the second unit of the neuron which contains the nonlinear activation function, in our model we use sigmoid function as our activation function [13]. The formula of sigmoid activation is: $f(x)=\frac{1}{1+e^{-i n p u t}}$

\subsection{The prediction phase}

When the neural network is trained then it is ready for prediction. After training with acceptable error the weights are set into the network then we give the trained network the input data set of the day which price we want to predict. The trained network then predicts the price using the given input data set.

\section{INPUT DATA}

Here is a brief description about the inputs that affect the share price:

\subsection{General Index (GI)}

General index is a number that measure the relative value of a section of share market. It reflects the total economic condition of the market. If the general index goes down then it means the economic condition of that particular market is relatively in poor condition.

\subsection{Net Asset Value (NAV)}

The Net asset value (NAV) of a company is the company's total assets minus its total liabilities. NAV is typically calculated on a per-share basis.

$\mathrm{NAV}=($ Net asset of a company - Liability $) /$ Total number of outstanding share

NAV is also calculated each day by taking the last market value of all securities owned plus all other assets such as cash, subtracting all liabilities and then dividing the result by the total number of shares outstanding.

NAV reflects the financial condition of the company. We can judge the company reputation by the NAV. 


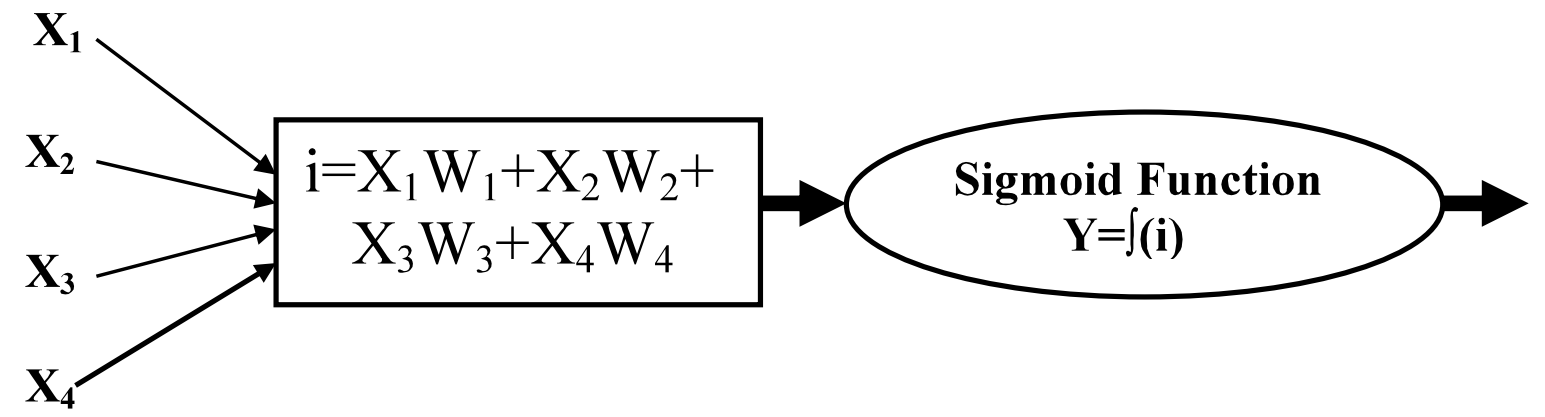

Fig 2: A Single Neuron

\subsection{P/E ratio}

The $\mathrm{P} / \mathrm{E}$ ratio makes a relationship between the share price and the company's earnings. The $\mathrm{P} / \mathrm{E}$ ratio of a share is a measure of the price paid for a share relative to the annual net income or profit earned by the firm per share.

$\mathrm{P} / \mathrm{E}$ ratio $=$ Share Price $/$ Earnings Per Share .

If $\mathrm{P} / \mathrm{E}$ ratio rises then there is a tendency of the company share price falls, the higher $\mathrm{P} / \mathrm{E}$ ratio then the higher probability to decrease the price.

\subsection{Earnings per Share (EPS)}

Earnings per share (EPS) is a comparison tool between two companies. Earnings per share serve as an indicator of a company's profitability.

EPS $=$ Net Earnings $/$ Number of Outstanding Shares.

For output we use the Price of the share. Using this data set we trained the network.

\subsection{Share Volume}

Share volume can be calculated in two different types the daily share volume and the monthly share volume. the total number of share is sold in a particular day is called daily share volume. In monthly share volume is the sum of the trading volumes during that month.

\section{SIMULATION PERFORMANCE ANALYSIS}

AND

Using the developed system to predict the future stock values with Feedforward Neural Networks we can do some analysis to know the performance of the Back-Propagation Algorithm.

By using the past historical data of ACI pharmaceutical company which include only 2 inputs, we tried to predict stock values for future 8 days of November 2010 from Back-Propagation algorithm we are now able to compare the predicted values with the real values. Table 1 and Figure 3 show the prediction and real values of the ACI pharmaceutical Company. The input past historical data is from 31-08-2010 to 30-09-2010.

The average error of the 1 st simulation was 3.71 percent.

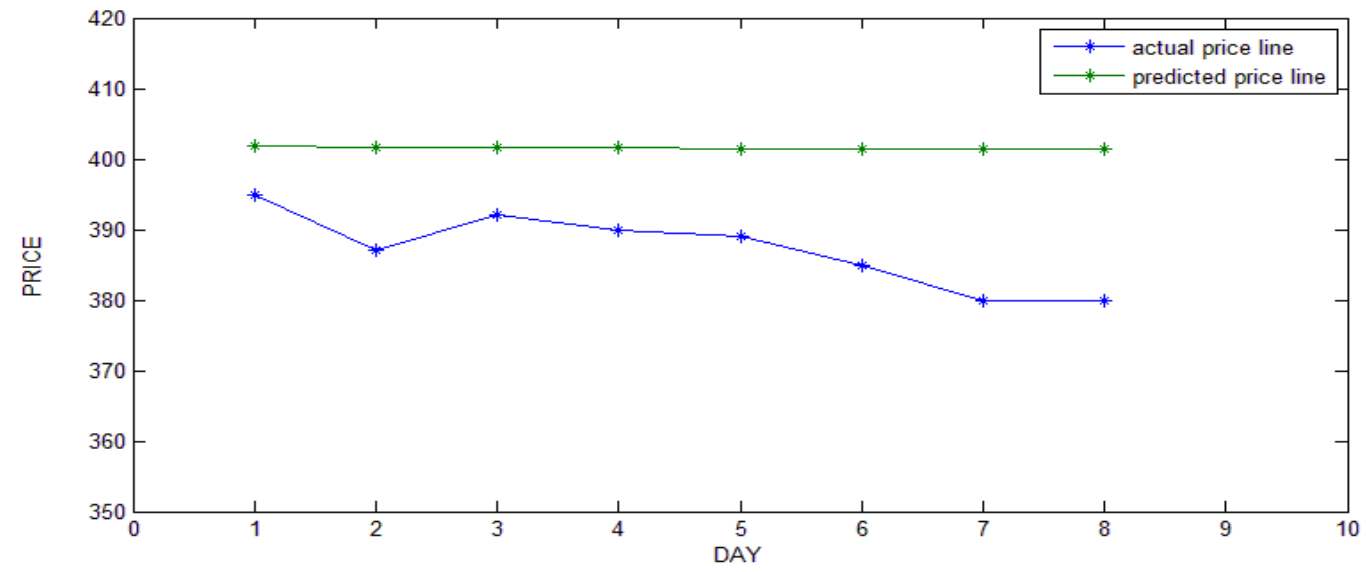

Fig - 3: Graphical representation of Predicting and Actual price of ACI pharmaceutical using 2 input data sets. 


\begin{tabular}{|c|l|l|l|}
\hline DATE & $\begin{array}{l}\text { Predicted } \\
\text { Price } \\
(\mathrm{TK})\end{array}$ & $\begin{array}{l}\text { Actual } \\
\text { Price } \\
(\mathrm{TK})\end{array}$ & $\begin{array}{l}\text { Error } \\
(\%)\end{array}$ \\
\hline 01-NOV-2010 & 401.9 & 395 & 1.74 \\
\hline 02-NOV-2010 & 401.7 & 387 & 3.79 \\
\hline 03-NOV-2010 & 401.7 & 392 & 2.47 \\
\hline 04-NOV-2010 & 401.7 & 390 & 3.00 \\
\hline 08-NOV-2010 & 401.5 & 389 & 3.21 \\
\hline 09-NOV-2010 & 401.4 & 385 & 4.24 \\
\hline 10-NOV-2010 & 401.5 & 380 & 5.65 \\
\hline 11-NOV-2010 & 401.5 & 380 & 5.65 \\
\hline
\end{tabular}

Table 1: Predicting price, Actual price and Error (\%) of ACI pharmaceutical using 2 input datasets.

For the second simulation we used the past historical data of ACI pharmaceutical company which include only 5 inputs, we tried to predict stock values for future 8 days of November. Table 2 and Figure 4 show the prediction and real values of the ACI pharmaceutical Company. The input past historical data is from 1-9-2010 to 31-10-2010. The average error of the $2^{\text {nd }}$ simulation was $1.53 \%$.

\begin{tabular}{|c|l|l|l|}
\hline DATE & $\begin{array}{l}\text { Predicted } \\
\text { Price } \\
(\mathrm{TK})\end{array}$ & $\begin{array}{l}\text { Actual } \\
\text { Price } \\
(\mathrm{TK})\end{array}$ & $\begin{array}{l}\text { Error } \\
(\%)\end{array}$ \\
\hline 01-NOV-2010 & 392.7 & 395 & 0.58 \\
\hline 02-NOV-2010 & 393.3 & 387 & 1.62 \\
\hline 03-NOV-2010 & 392.5 & 392 & 0.12 \\
\hline 04-NOV-2010 & 392.0 & 390 & 0.51 \\
\hline 08-NOV-2010 & 392.8 & 389 & 0.97 \\
\hline 09-NOV-2010 & 392.5 & 385 & 1.94 \\
\hline 10-NOV-2010 & 392.5 & 380 & 3.28 \\
\hline 11-NOV-2010 & 392.3 & 380 & 3.23 \\
\hline
\end{tabular}

Table 2: Predicting price, Actual price and Error (\%) of ACI pharmaceutical using 5 input data sets.

The more input data we have the better training and get more close results. This means that, more the available data for predicting financial markets, the greater the chances of an accurate forecast. But the sum squared error was high for the 5 input dataset than the 2 input data sets but the prediction error was minimal.

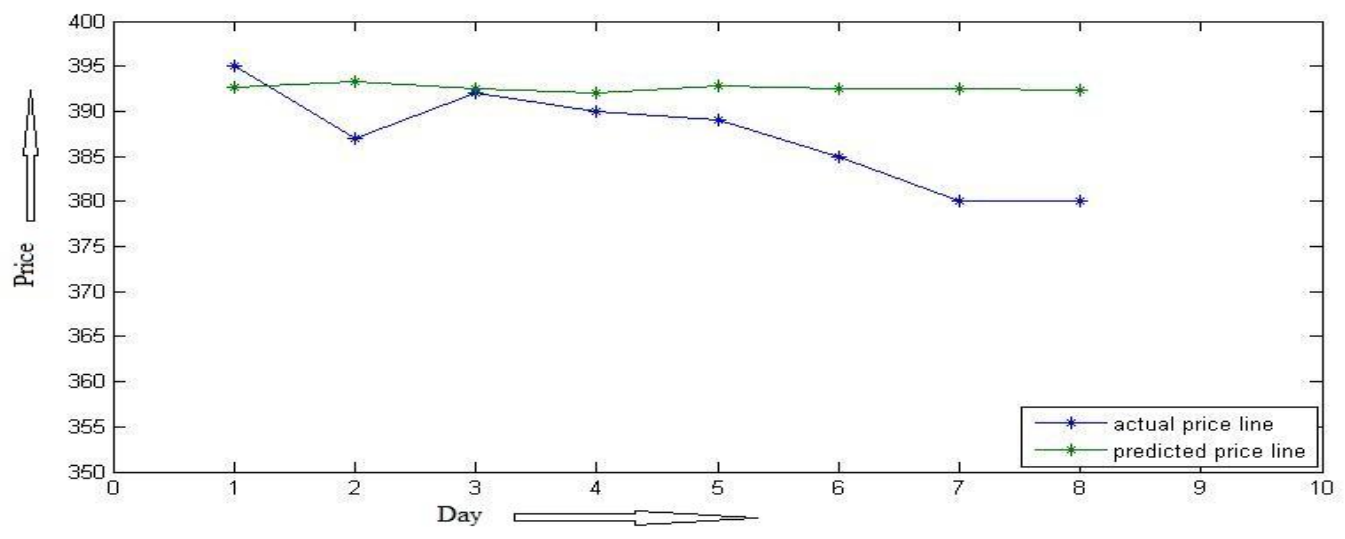

Fig - 4: Graphical representation of Predicting and Actual price of ACI pharmaceutical using 5 input data sets.

\subsection{Observation}

1. We observed that when we take 2 inputs for prediction the sum squared error was high. But when we take 4 inputs the sum squared error was minimized. But when we take 5 input then the sum squared error is higher than the 4 input technique.

2. When we take the data of share market in a sequential date, we can predict the share price nearer to the actual price. But if we take the data of discontinuous date the difference between the predicted price and actual price was relatively high.

3. In the training time if any input changed suddenly at a high rate then the prediction was not near to the actual price.

\section{CONCLUSION}

As researchers and investors strive to out-perform the market, the use of neural networks to forecast stock market prices will be a continuing area of research. The ultimate goal is to increase the yield from the investment. It has been proven already through research that the evaluation of the return on investment in share markets through any of the traditional techniques is tedious, expensive and a timeconsuming process. In conclusion we can say that if we train our system with more input data set it generate more error free prediction price. 


\section{REFERENCES}

[1] Z.Tang and P.A.Fishwick, "Backpropagation neural nets as models for time series forcasting," ORSA journal on computing, vol.5, No. 4, pp 374-384, 1993.

[2] J.H.wang and J.Y.Leu, "stock market trend prediction using ARIMA-based neural network,"Proc. Of IEEE conference on neural networks, vol.4, pp.2160-2165, 1996

[3] Kimoto,T., asakawa, K., Yoda , M,. and Takeoka, M. (1990),Stock market prediction system with modular neural network, in proceedings of the International Joint Conference on Neural Network, 1-6.

[4] Mizuno, H., Kosaka , M., Yajima , H. and Komoda N. (1998), Application of Neural Network to Technical Analysis of Stock Market Prediction, Studies in Information and Control, vol.7, no.3, pp.111-120.

[5] Sexton, R. S., R. E. Dorsey and J.D.Dohnson (1998), Toward global optimization of neural networks: A comparison of the genetic algorithm and backpropagation, Decision Support systems 22, 171185 .

[6] Phua, P.K.H. Ming, D., Lin, W. (2000), Neural network With Genetic Algorithms For Stocks Predictio, Fifth Conference of the Association of Asian-Pacific Operations Research Societies, $5^{\text {th }}-7^{\text {th }}$ july, Singapore.

[7] Samarth Agarwal,Manol Jindal,G.N.Pillai "Momentum Analysis based Stock Market Prediction using
ANFIS". In Proceeding of the International Multiconference of Engineering and Computer Scientists 2010 Vol.1, IMECS 2010, March 2010, Hong Kong.

[8] Rumelhart, D.D.m Hinton, G.E. and Williams, R.J., Learning Internal Representation, Man, and Cybernetics (SMC'91), 1991. 1913-1918.

[9] "Feedforward neural Networks: An Introduction" by Simo Haykin page (2-4)

[10] "Artificial Intelligence a morden approach" (second edition) by Stuart Russell, Peter Norvig 2004

[11] Simon Haykin, "Neural Network A Comprehensive Foundation", second edition, Prentice Hall, 1998, page $161-173$

[12] Robert J. Van Eyden. "The Application of Neural Networks in the Forcasting of Share Prices". Finance and Technology Publishing, 1996.

[13] W.Duch and N. Jankowski, "Transfer functions: hidden possibilities for better neural networks.", 9th European Symposium on Artificial Neural Networks (ESANN), Brugge 2001. De-facto publications.

[14] Y.-Q. Zhang and A. Kandel, "Compensatory Genetic Fuzzy Neural Networks and Their Applications," Series in Machine Perception Artificial Intelligence, Volume 30, World Scientific, 1998. 